\title{
Autologous mDC3 Vaccine
}

National Cancer Institute

\section{Source}

National Cancer Institute. Autologous mDC3 Vaccine. NCI Thesaurus. Code C162250.

An antineoplastic vaccine composed of autologous mature dendritic cells (mDCs) pulsed

with mutated peptides, with potential immunostimulating and antineoplastic activities.

Upon administration, the autologous mDC3 vaccine may elicit a cytotoxic T-cell (CTL)

response against cancer cells. 